\title{
A subclass of univalent functions associated with $q$-analogue of Choi-Saigo-Srivastava operator
}

\author{
Zhi-Gang Wang*1 (D), S. Hussain² (D), M. Naeem³ (D), T. Mahmood ${ }^{3}$ (D), S. $\operatorname{Khan}^{4}$ (D) \\ ${ }^{1}$ School of Mathematics and Computing Science, Hunan First Normal University, Changsha 410205, \\ Hunan, People's Republic of China \\ ${ }^{2}$ Department of Mathematics, Comsats University Islamabad, Abbottabad Campus 22010, Pakistan \\ ${ }^{3}$ Department of Mathematics and Statistics, International Islamic University, Islamabad 44000, Pakistan \\ ${ }^{4}$ Department of Mathematics, Riphah International University, Islamabad 44000, Pakistan
}

\begin{abstract}
The main objective of the present paper is to define a subclass $Q_{q}(\lambda, \mu, A, B)$ of analytic functions by using subordination along with the newly defined $q$-analogue of Choi-SaigoSrivastava operator. Such results as coefficient estimates, integral representation, linear combination, weighted and arithmetic means, and radius of starlikeness for this class are derived.
\end{abstract}

Mathematics Subject Classification (2010). 30C45, 30C50

Keywords. analytic functions, univalent functions, $q$-differential operator, $q$-analogue of Choi-Saigo-Srivastava operator

\section{Introduction}

Let $\mathbb{E}=\{z \in \mathbb{C}:|z|<1\}$ be the open unit disk and $\mathcal{A}$ be the class of all functions $f$ which are analytic in $\mathbb{E}$ and normalized by $f(0)=0$ and $f^{\prime}(0)=1$. Thus, each $f \in \mathcal{A}$ has the Maclaurin's series expansion of the form:

$$
f(z)=z+\sum_{n=2}^{\infty} a_{n} z^{n}
$$

For two functions $f$ and $g$ analytic in $\mathbb{E}$, we say that $f$ is subordinate to $g$, written by $f(z) \prec g(z)$, if there exists an analytic function $\omega(z)$ with $\omega(0)=0$ and $|\omega(z)|<1$ such that $f(z)=g(\omega(z))$. In particular, if $g$ is univalent in $\mathbb{E}$, then $f(0)=g(0)$ and $f(\mathbb{E}) \subset g(\mathbb{E})$. For two functions $f$ of the form (1.1) and $g$ of the form

$$
g(z)=z+\sum_{n=2}^{\infty} b_{n} z^{n}
$$

\footnotetext{
* Corresponding Author.

Email addresses: zhigangwang@foxmail.com (Z.-G. Wang), saqib_math@yahoo.com (S. Hussain), naeem.phdma75@iiu.edu.pk (M. Naeem), tahirbakhat@iiu.edu.pk (T. Mahmood),

shahidmath761@gmail.com (S. Khan)

Received: 12.06.2019; Accepted: 09.11.2019
} 
that are analytic in $\mathbb{E}$, we define the convolution of these functions by

$$
(f * g)(z)=z+\sum_{k=2}^{\infty} a_{n} b_{n} z^{n} .
$$

Many differential and integral operators can be written in terms of convolution; we refer to $[2-4,6,10,19]$. It is worth mentioning that the technique of convolution helps researchers in further investigation of geometric properties of analytic functions.

Let $\mathcal{S} \subset \mathcal{A}$ be the class of functions which are univalent in $\mathbb{E}$. A function $f \in \mathcal{A}$ is in the class $\mathcal{S}^{*}(\gamma)$ of starlike function of order $\gamma$, if

$$
\Re\left(\frac{z f^{\prime}(z)}{f(z)}\right)>\gamma \quad(0 \leq \gamma<1) .
$$

We note that $\mathcal{S}^{*}(0)=\mathcal{S}^{*}$, the familiar class of starlike functions. An analytic function $h$ with $h(0)=1$ is said to be in the Janowski class $\mathcal{P}[A, B]$, if and only if

$$
h(z) \prec \frac{1+A z}{1+B z} \quad(-1 \leq B<A \leq 1) .
$$

The class $\mathcal{P}[A, B]$ of Janowski functions was introduced by Janowski [15,24].

Recently, the study of $q$-analysis ( $q$-calculus) has inspired the researchers due to its applications in mathematics and other related areas. Jackson $[13,14]$ had defined the $q$ analogue of derivative and integral operator as well as provided some of their applications. Later, Aral and Gupta [8,9] introduced the $q$-Baskakov-Durrmeyer operator by using $q$ beta function, while the authors of $[5,7]$ studied the $q$-generalization of complex ope-

rators known as $q$-Picard and $q$-Gauss-Weierstrass singular integral operators. Recently, Kanas and Raducanu [16] introduced the $q$-analogue of Ruscheweyh differential operator by using the concept of convolution and studied some of its properties. Aldweby and Darus [1], Mahmood and Sokol [18] studied some classes of analytic functions defined by means of $q$-analogue of Ruscheweyh differential operator. Many $q$-differential and $q$ integral operators can be written in terms of convolution, for details see $[11,12,22,23,25]$. The current paper aims to express a $q$-analogue of Choi-Saigo-Srivastava operator involving convolution concepts. Besides, it also aims to give some interesting applications of this operator. Here we will present the basic concept of $q$-calculus which was initiated by Jackson [14] will help us in further study. Furthermore, such approach can be generalized to domains in higher dimensions.

For $0<q<1$, the $q$-derivative of a function $f$ is defined by

$$
\partial_{q} f(z)=\frac{f(q z)-f(z)}{z(q-1)} .
$$

It can easily be seen that for $n \in \mathbb{N}:=\{1,2, \cdots\}$ and $z \in \mathbb{E}$,

$$
\partial_{q}\left\{\sum_{n=1}^{\infty} a_{n} z^{n}\right\}=\sum_{n=1}^{\infty}[n, q] a_{n} z^{n-1},
$$

where

$$
[n, q]=\frac{1-q^{n}}{1-q}=1+\sum_{l=1}^{n-1} q^{l}, \quad[0, q]=0 .
$$

For any non-negative integer $n$, the $q$-number shift factorial is defined by

$$
[n, q] != \begin{cases}1 & (n=0), \\ {[1, q][2, q][3, q] \cdots[n, q]} & (n \in \mathbb{N}) .\end{cases}
$$

Also the $q$-generalized Pochhammer symbol for $x>0$ is given by

$$
[x, q]_{n}= \begin{cases}1 & (n=0), \\ {[x, q][x+1, q] \cdots[x+n-1, q]} & (n \in \mathbb{N}),\end{cases}
$$


and for $x>0$, let $q$-gamma function be defined by

$$
\Gamma_{q}(x+1)=[x, q] \Gamma_{q}(t) \text { and } \Gamma_{q}(1)=1 .
$$

Using the definition of $q$-derivative along with the idea of convolution, we now define the $q$-Choi-Saigo-Srivastava operator as:

$$
I_{\lambda, \mu}^{q} f(z)=f(z) * \mathcal{F}_{q, \lambda+1, \mu}(z) \quad(z \in \mathbb{E} ; \lambda>-1 ; \mu>0 ; f \in \mathcal{A}),
$$

where

$$
\mathcal{F}_{q, \lambda+1, \mu}(z)=z+\sum_{n=2}^{\infty} \frac{\Gamma_{q}(\mu+n-1) \Gamma_{q}(1+\lambda)}{\Gamma_{q}(\mu) \Gamma_{q}(n+\lambda)} z^{n}=z+\sum_{n=2}^{\infty} \frac{[\mu, q]_{n-1}}{[1+\lambda, q]_{n-1}} z^{n} .
$$

Thus, we see that

$$
I_{\lambda, \mu}^{q} f(z)=z+\sum_{n=2}^{\infty} \frac{[\mu, q]_{n-1}}{[1+\lambda, q]_{n-1}} a_{n} z^{n}
$$

Clearly,

$$
I_{0,2}^{q} f(z)=z \partial_{q} f(z) \text { and } I_{1,2}^{q} f(z)=f(z) .
$$

From (1.5), we can easily get the identities

$$
[\lambda+1, q] I_{\lambda, \mu}^{q} f(z)=q^{\lambda} z \partial_{q}\left(I_{\lambda+1, \mu}^{q} f(z)\right)+[\lambda, q] I_{\lambda+1, \mu}^{q} f(z),
$$

and

$$
q^{\lambda} z \partial_{q}\left(I_{\lambda, \mu}^{q} f(z)\right)=[\mu, q] I_{\lambda, \mu+1}^{q} f(z)-([\mu-1, q]) I_{\lambda, \mu}^{q} f(z) .
$$

If $q \rightarrow 1$, the relationships (1.6) and (1.7) imply that

$$
z\left(I_{\lambda+1} f(z)\right)^{\prime}=(1+\lambda) I_{\lambda, \mu} f(z)-\lambda I_{\lambda+1, \mu} f(z),
$$

and

$$
z\left(I_{\lambda, \mu} f(z)\right)^{\prime}=\mu I_{\lambda, \mu+1} f(z)-(\mu-1) I_{\lambda+1, \mu} f(z),
$$

which are the well-known identities associated with Choi-Saigo-Srivastava operator. By taking specific values of parameters, we obtain various known operators studied earlier in the literature.

(1) For $\mu=2$, we obtain $q$-analogue of Noor integral operator studied in [27], which is defined as:

$$
I_{\lambda, 2}^{q} f(z)=z+\sum_{n=2}^{\infty} \frac{[n, q] !}{[1+\lambda, q]_{n-1}} a_{n} z^{n}
$$

(2) For $\mu=2$ and $q \rightarrow 1$, we get the differential operator studied in [20,21], which is defined as:

$$
I^{n} f(z)=z+\sum_{n=2}^{\infty} \frac{n !}{(1+\lambda)_{n-1}} a_{n} z^{n}
$$

(3) For $\mu=2, \lambda=1-\alpha$, and $q \rightarrow 1$, we obtain Owa-Srivastava operator studied in [26], which is defined as:

$$
I_{1-\alpha, 2} f(z)=z+\sum_{n=2}^{\infty} \frac{\Gamma(n+1) \Gamma(2-\alpha)}{\Gamma(n+1-\alpha)} a_{n} z^{n} .
$$

In this paper, we aim to investigate the following subclass of analytic functions associated with the operator $I_{\lambda, \mu}^{q}$. 
Definition 1.1. Let $-1 \leq B<A \leq 1$ and $0<q<1$. The function $f \in \mathcal{A}$ is in the class $Q_{q}(\lambda, \mu, A, B)$ if it satisfies

$$
\frac{z \partial_{q}\left(I_{\lambda, \mu}^{q} f(z)\right)}{I_{\lambda, \mu}^{q} f(z)} \prec \frac{1+A z}{1+B z} .
$$

Equivalently, a function $f \in Q_{q}(\lambda, \mu, A, B)$ if and only if

$$
\left|\frac{\frac{z \partial_{q}\left(I_{\lambda, \mu}^{q} f(z)\right)}{I_{\lambda, \mu}^{q} f(z)}-1}{A-B\left(\frac{z \partial_{q}\left(I_{\lambda, \mu}^{q} f(z)\right)}{I_{\lambda, \mu}^{q} f(z)}\right)}\right|<1
$$

We need the following lemma to prove one of our result.

Lemma 1.2. [17] Let $-1 \leq B_{2} \leq B_{1}<A_{1} \leq A_{2} \leq 1$. Then

$$
\frac{1+A_{1} z}{1+B_{1} z} \prec \frac{1+A_{2} z}{1+B_{2} z} .
$$

Throughout this paper, we assume that $\lambda>-1, \mu>0,0<q<1$ and $-1 \leq B<A \leq 1$, unless otherwise stated. We also suppose that all coefficients $a_{n}$ of $f$ are real positive numbers.

\section{Main results}

Theorem 2.1. Let $f \in \mathcal{A}$ and be of the form (1.1). Then $f \in Q_{q}(\lambda, \mu, A, B)$ if and only if

$$
\sum_{n=2}^{\infty}\{[n, q](1-B)-1+A\} \frac{[\mu, q]_{n-1}}{[1+\lambda, q]_{n-1}} a_{n}<A-B .
$$

Proof. Assume that (2.1) holds. To show that $f \in Q_{q}(\lambda, \mu, A, B)$, we only need to prove the inequality (1.8). For this, we consider

$$
\begin{aligned}
\left|\frac{\frac{z \partial_{q}\left(I_{\lambda, \mu}^{q} f(z)\right)}{I_{\lambda, \mu}^{q} f(z)}-1}{A-B\left(\frac{z \partial_{q}\left(I_{\lambda, \mu}^{q} f(z)\right)}{I_{\lambda, \mu}^{q} f(z)}\right)}\right| & =\left|\frac{\sum_{n=2}^{\infty}([n, q]-1) \frac{[\mu, q]_{n-1}}{[1+\lambda, q] n-1} a_{n} z^{n}}{(A-B) z+\sum_{n=2}^{\infty}\{A-B[n, q]\} \frac{[\mu, q]_{n-1}}{[1+\lambda, q]_{n-1}} a_{n} z^{n}}\right| \\
& \leq \frac{\sum_{n=2}^{\infty}([n, q]-1) \frac{[\mu, q]_{n-1}}{[1+\lambda, q]_{n-1}} a_{n}}{(A-B)-\sum_{n=2}^{\infty}\{A-B[n, q]\} \frac{[\mu, q]_{n-1}}{[1+\lambda, q]_{n-1}} a_{n}}<1
\end{aligned}
$$

where we have used (1.2), (1.5), and (2.1) and this completes the direct part.

Conversely, let $f \in Q_{q}(\lambda, \mu, A, B)$ be of the form (1.1), then from (1.8) along with (1.5), we have

$$
\left|\frac{\frac{z \partial_{q}\left(I_{\lambda, \mu}^{q} f(z)\right)}{I_{\lambda, \mu}^{q} f(z)}-1}{A-B\left(\frac{z \partial_{q}\left(I_{\lambda, \mu}^{q} f(z)\right)}{I_{\lambda, \mu}^{q} f(z)}\right)}\right|=\left|\frac{\sum_{n=2}^{\infty}([n, q]-1) \frac{[\mu, q]_{n-1}}{[1+\lambda, q]_{n-1}} a_{n} z^{n}}{(A-B) z+\sum_{n=2}^{\infty}\{A-B[n, q]\} \frac{[\mu, q]_{n-1}}{[1+\lambda, q]_{n-1}} a_{n} z^{n}}\right|<1 .
$$

Since $|\Re(z)| \leq|z|$, we get

$$
\Re\left(\frac{\sum_{n=2}^{\infty}([n, q]-1) \frac{[\mu, q]_{n-1}}{[1+\lambda, q]_{n-1}} a_{n} z^{n}}{(A-B)+\sum_{n=2}^{\infty}\{A-B[n, q]\} \frac{[\mu, q]_{n-1}}{[1+\lambda, q]_{n-1}} a_{n} z^{n}}\right)<1 .
$$

Now, we choose values of $z$ on the real axis such that $\frac{z \partial_{q}\left(I_{\lambda, \mu}^{q} f(z)\right)}{I_{\lambda, \mu}^{q} f(z)}$ is real. Upon clearing the denominator in (2.2) and letting $z \rightarrow 1^{-}$through real values, we obtain the required inequality (2.1). 
Theorem 2.2. Let $f \in Q_{q}(\lambda, \mu, A, B)$. Then

$$
I_{\lambda, \mu}^{q} f(z)=\exp \left(\int_{0}^{z} \frac{1}{t}\left(\frac{1-A \phi(t)}{1-B \phi(t)}\right) d_{q}(t)\right),
$$

where $|\phi(z)|<1$.

Proof. Let $f \in Q_{q}(\lambda, \mu, A, B)$ and setting

$$
\frac{z \partial_{q} I_{\lambda, \mu}^{q} f(z)}{I_{\lambda, \mu}^{q} f(z)}=h(z)
$$

with

equivalently, we can write

$$
h(z) \prec \frac{1+A z}{1+B z},
$$

then we have

$$
\left|\frac{h(z)-1}{A-B h(z)}\right|<1
$$

$$
\frac{h(z)-1}{A-B h(z)}=\phi(z)
$$

where $|\phi(z)|<1$. Thus, we can rewrite

$$
\frac{\partial_{q}\left(I_{\lambda, \mu}^{q} f(z)\right)}{I_{\lambda, \mu}^{q} f(z)}=\frac{1}{z}\left(\frac{1-A \phi(t)}{1-B \phi(t)}\right) .
$$

By simple computation along with integration, we obtain the required result.

Theorem 2.3. Let $f_{j} \in Q_{q}(\lambda, \mu, A, B)$ and have the form

$$
f_{j}(z)=z+\sum_{k=1}^{\infty} a_{k, j} z^{k} \quad(j=1,2,3, \ldots, l) .
$$

Then $F \in Q_{q}(\lambda, \mu, A, B)$, where

$$
F(z)=\sum_{j=1}^{l} c_{j} f_{j}(z) \text { with } \sum_{j=1}^{l} c_{j}=1 .
$$

Proof. By the virtue of Theorem 2.1, one can write

$$
\sum_{n=2}^{\infty}\left\{\frac{\{[n, q](1-B)-1+A\} \frac{[\mu, q]_{n-1}}{[1+\lambda, q]_{n-1}}}{A-B}\right\} a_{n, j}<1 .
$$

Therefore, we obtain

$$
F(z)=\sum_{j=2}^{l} c_{j}\left(z+\sum_{n=2}^{\infty} a_{n, j} z^{n}\right)=z+\sum_{j=2}^{l} \sum_{n=2}^{\infty} c_{j} a_{n, j} z^{n}=z+\sum_{n=2}^{\infty}\left(\sum_{j=2}^{l} c_{j} a_{n, j}\right) z^{n} .
$$

However,

$$
\begin{aligned}
& \sum_{n=2}^{\infty} \frac{\{[n, q](1-B)-1+A\} \frac{[\mu, q]_{n-1}}{[1+\lambda, q]_{n-1}}}{A-B}\left(\sum_{j=2}^{l} a_{n . j} c_{j}\right) \\
= & \sum_{j=2}^{l}\left\{\sum_{n=2}^{\infty} \frac{\{[n, q](1-B)-1+A\} \frac{[\mu, q]_{n-1}}{[1+\lambda, q]_{n-1}}}{A-B} a_{n . j}\right\} c_{j} \leq 1,
\end{aligned}
$$

then $F \in Q_{q}(\lambda, \mu, A, B)$. Hence the proof is completed. 
Theorem 2.4. If $f$ and $g$ belong to $Q_{q}(\lambda, \mu, A, B)$, then their weighted mean $h_{j}(j \in \mathbb{N})$ is also in $Q_{q}(\lambda, \mu, A, B)$, where $h_{j}$ is defined by

$$
h_{j}(z)=\frac{(1-j) f(z)+(1+j) g(z)}{2} .
$$

Proof. From (2.3), we can write

$$
h_{j}(z)=z+\sum_{n=2}^{\infty}\left\{\frac{(1-j) a_{n}+(1+j) b_{n}}{2}\right\} z^{n} .
$$

To prove $h_{j}(z) \in Q_{q}(\lambda, \mu, A, B)$, we need to show that

$$
\sum_{n=2}^{\infty} \frac{\{[n, q](1-B)-1+A\}}{A-B}\left\{\frac{(1-j) a_{n}+(1+j) b_{n}}{2}\right\} \frac{[\mu, q]_{n-1}}{[1+\lambda, q]_{n-1}}<1 .
$$

For this, consider

$$
\begin{aligned}
& \sum_{n=2}^{\infty} \frac{\{[n, q](1-B)-1+A\}}{A-B}\left\{\frac{(1-j) a_{n}+(1+j) b_{n}}{2}\right\} \frac{[\mu, q]_{n-1}}{[1+\lambda, q]_{n-1}} \\
= & \frac{(1-j)}{2} \sum_{n=2}^{\infty} \frac{\{[n, q](1-B)-1+A\}}{A-B} \frac{[\mu, q]_{n-1}}{[1+\lambda, q]_{n-1}} a_{n} \\
& +\frac{(1+j)}{2} \sum_{n=2}^{\infty} \frac{\{[n, q](1-B)-1+A\}}{A-B} \frac{[\mu, q]_{n-1}}{[1+\lambda, q]_{n-1}} b_{n} \\
< & \frac{(1-j)}{2}+\frac{(1+j)}{2}=1,
\end{aligned}
$$

where we have used the inequality (2.1). Hence the result follows.

Theorem 2.5. Let $f_{j}$ with $j=1,2, \ldots, \alpha(\alpha \in \mathbb{N})$ belong to the class $Q_{q}(\lambda, \mu, A, B)$. Then the arithmetic mean $h$ of $f_{j}$ given by

$$
h(z)=\frac{1}{\alpha} \sum_{j=1}^{\alpha} f_{j}(z)
$$

also belongs to the class $Q_{q}(\lambda, \mu, A, B)$.

Proof. From (2.4), we can write

$$
h(z)=\frac{1}{\alpha} \sum_{j=1}^{\alpha}\left(z+\sum_{n=2}^{\infty} a_{n, j} z^{n}\right)=z+\sum_{n=2}^{\infty}\left(\frac{1}{\alpha} \sum_{j=1}^{\alpha} a_{n, j}\right) z^{n} .
$$

Since $f_{j} \in Q_{q}(\lambda, \mu, A, B)$, for every $j=1,2, \ldots, \alpha$, by means of (2.5) and (2.1), we have

$$
\begin{aligned}
& \sum_{n=2}^{\infty}\{[n, q](1-B)-1+A\} \frac{[\mu, q]_{n-1}}{[1+\lambda, q]_{n-1}}\left(\frac{1}{\alpha} \sum_{j=1}^{\alpha} a_{n, j}\right) \\
= & \frac{1}{\alpha} \sum_{j=1}^{\alpha}\left(\sum_{n=2}^{\infty}\{[n, q](1-B)-1+A\} \frac{[\mu, q]_{n-1}}{[1+\lambda, q]_{n-1}} a_{n, j}\right) \\
\leq & \frac{1}{\alpha} \sum_{j=1}^{\alpha}(A-B)=A-B,
\end{aligned}
$$

and this completes the proof. 
Theorem 2.6. Let $f \in Q_{q}(\lambda, \mu, A, B)$. Then $f \in \mathcal{S}^{*}(\gamma)$, for $|z|<r_{1}$, where

$$
r_{1}=\left(\frac{(1-\gamma)\{[n, q](1-B)-1+A\} \frac{[\mu, q]_{n-1}}{[1+\lambda, q]_{n-1}}}{(n-\gamma)(A-B)}\right)^{\frac{1}{n-1}} .
$$

Proof. Let $f \in Q_{q}(\lambda, \mu, A, B)$. To prove $f \in \mathcal{S}^{*}(\gamma)$, we only need to show that

$$
\left|\frac{\frac{z f^{\prime}(z)}{f(z)}-1}{\frac{z f^{\prime}(z)}{f(z)}+1-2 \gamma}\right|<1
$$

By using (1.1) along with some simple computations we have

$$
\sum_{n=2}^{\infty}\left(\frac{n-\gamma}{1-\gamma}\right)\left|a_{n}\right||z|^{n-1}<1
$$

Since $f \in Q_{q}(\lambda, \mu, A, B)$, from (2.1), we can easily obtain

$$
\sum_{n=2}^{\infty} \frac{\{[n, q](1-B)-1+A\} \frac{[\mu, q]_{n-1}}{[1+\lambda, q]_{n-1}}}{A-B}\left|a_{n}\right|<1 .
$$

Now, the inequality (2.6) is true, if the following inequality

$$
\sum_{n=2}^{\infty}\left(\frac{n-\gamma}{1-\gamma}\right)\left|a_{n}\right||z|^{n-1}<\sum_{n=2}^{\infty} \frac{\{[n, q](1-B)-1+A\} \frac{[\mu, q]_{n-1}}{[1+\lambda, q]_{n-1}}}{A-B}\left|a_{n}\right|
$$

holds, which implies that

$$
|z|^{n-1}<\frac{(1-\gamma)\{[n, q](1-B)-1+A\} \frac{[\mu, q]_{n-1}}{[1+\lambda, q]_{n-1}}}{(A-B)(n-\gamma)},
$$

and thus we get the required result.

Theorem 2.7. Let $-1 \leq B_{2} \leq B_{1}<A_{1} \leq A_{2} \leq 1$ and $I_{\lambda+1, \mu}^{q} f(z) \neq 0$ in $\mathbb{E}$. If

$$
\frac{([\lambda+1, q]) I_{\lambda, \mu}^{q} f(z)}{q^{\lambda} I_{\lambda+1, \mu}^{q} f(z)}-\frac{[\lambda, q]}{q^{\lambda}} \prec \frac{1+A_{1} z}{1+B_{1} z} .
$$

Then $f \in Q_{q}\left(\lambda+1, \mu, A_{2}, B_{2}\right)$.

Proof. Since $I_{\lambda+1, \mu}^{q} f(z) \neq 0$ in $\mathbb{E}$, we define the function $p(z)$ by

$$
\frac{z \partial_{q}\left(I_{\lambda+1, \mu}^{q} f(z)\right)}{I_{\lambda+1, \mu}^{q} f(z)}=p(z) .
$$

By virtue of (1.6), we obtain

$$
\frac{([\lambda+1, q]) I_{\lambda, \mu}^{q} f(z)}{q^{\lambda} I_{\lambda+1, \mu}^{q} f(z)}-\frac{[\lambda, q]}{q^{\lambda}}=p(z) .
$$

Therefore, from (2.8), we have

$$
\frac{z \partial_{q}\left(I_{\lambda+1, \mu}^{q} f(z)\right)}{I_{\lambda+1, \mu}^{q} f(z)}=p(z) \prec \frac{1+A_{1} z}{1+B_{1} z},
$$

by Lemma 1.2 , we deduce that $f \in Q_{q}\left(\lambda+1, \mu, A_{2}, B_{2}\right)$.

Acknowledgment. The present investigation was supported by the Key Project of Education Department of Hunan Province under Grant no. 19A097 of the People's Republic of China. The authors would like to thank the referees for their valuable comments and suggestions, which was essential to improve the quality of this paper. 


\section{References}

[1] H. Aldweby and M. Darus, Some subordination results on q-analogue of Ruscheweyh differential operator, Abstr. Appl. Anal. 2014, 1-9, 2014.

[2] F.M. Al-Oboudi, On univalent functions defined by a generalized Salagean operator, Int. J. Math. Math. Sci. 2004, 1419-1436, 2004.

[3] F.M. Al-Oboudi, On classes of functions related to starlike functions with respect to symmetric conjugate points defined by a fractional differential operator, Complex Anal. Oper. Theory, 5, 647-658, 2011.

[4] F.M. Al-Oboudi and K.A. Al-Amoudi, On classes of analytic functions related to conic domains, J. Math. Anal. Appl. 399, 655-667, 2008.

[5] G.A. Anastassiou and S.G. Gal, Geometric and approximation properties of generalized singular integrals in the unit disk, J. Korean Math. Soc. 23, 425-443, 2006.

[6] A. Aral, On the generalized Picard and Gauss Weierstrass singular integrals, J. Comput. Anal. Appl. 8, 249-261, 2006.

[7] A. Aral, On the generalized Picard and Gauss Weierstrass singular integrals, J. Comput. Anal. Appl. 8, 249-261, 2006.

[8] A. Aral and V. Gupta, On q-Baskakov type operators, Demonstr. Math. 42, 109-122, 2009.

[9] A. Aral and V. Gupta, Generalized q-Baskakov operators, Math. Slovaca, 61, 619-634, 2011.

[10] S.Z.H. Bukhari, M. Nazir, and M. Raza, Some generalisations of analytic functions with respect to $2 k$-symmetric conjugate points, Maejo Int. J. Sci. Technol. 10, 1-12, 2016.

[11] M. Govindaraj and S. Sivasubramanian, On a class of analytic functions related to conic domains involving q-calculus, Anal. Math. 43, 475-487, 2017.

[12] S. Hussain, S. Khan, M.A. Zaighum, and M. Darus, Applications of a q-Salagean type operator on multivalent functions, J. Inequal. Appl. 2018, Art. 301, 2018.

[13] F.H. Jackson, On q-functions and a certain difference operator, Earth Environ. Sci. Tran. R. Soc. Edinb. 46, 253-281, 1909.

[14] F.H. Jackson, On q-definite integrals, Quart. J. Pure Appl. Math. 41, 193-203, 1910.

[15] W. Janowski, Some extremal problems for certain families of analytic functions, Ann. Polon. Math. 28, 297-326, 1973.

[16] S. Kanas and D. Raducanu, Some class of analytic functions related to conic domains, Math. Slovaca, 64, 1183-1196, 2014.

[17] M.-S. Liu, On a subclass of p-valent close to convex functions of type $\alpha$ and order $\beta$, J. Math. Study 30, 102-104, 1997.

[18] S. Mahmood and J. Sokol, New subclass of analytic functions in conical domain associated with Ruscheweyh q-differential operator, Results Math. 71, 1-13, 2017.

[19] M. Naeem, S. Hussain, T. Mahmood, S. Khan, and M. Darus, A new subclass of analytic functions defined by using Salagean q-differential operator, Mathematics, 7, 458-469, 2019.

[20] K.I. Noor, On new classes of integral operator, J. Natur. Geom. 16, 71-80, 1999.

[21] K.I. Noor and M. A. Noor, On integral operators, J. Math. Anal. Appl. 238, 341-352, 1999.

[22] K.I. Noor, N. Khan, and Q.Z. Ahmad, Some properties of multivalent spiral-like functions, Maejo Int. J. Sci. Technol. 12, 139-151, 2018.

[23] M. Sabil, Q.Z. Ahmad, B. Khan, M. Tahir, and N. Khan, Generalisation of certain subclasses of analytic and bi-univalent functions, Maejo Int. J. Sci. Technol. 13, 1-9, 2019 . 
[24] F.M. Sakar and S.M. Aydoğan, Subclass of m-quasiconformal harmonic functions in association with Janowski starlike functions, Appl. Math. Comput. 319, 461-468, 2018.

[25] F.M. Sakar and S.M. Aydoğan, Coefficient bounds for certain subclasses of $m$-fold symmetric bi-univalent functions defined by convolution, Acta Univ. Apulensis Math. Inform. 55, 11-21, 2018.

[26] Z. Shareef, S. Hussain, and M. Darus, Convolution operator in geometric functions theory, J. Inequal. Appl. 2012, Art. 213, 2012.

[27] H.M. Srivastava, S. Khan, Q.Z. Ahmad, N. Khan, and S. Hussain, The Faber polynomial expansion method and its application to the general coefficient problem for some subclasses of bi-univalent functions associated with a certain q-integral operator, Stud. Univ. Babes-Bolyai Math. 63, 419-436, 2018. 